\title{
Bioinspired non-invasive radial pulse sensor: from biomimetic design, system calibration, to clinic application
}

\author{
Yudong Luo ${ }^{1}$, Sanku Niu ${ }^{1,2}$, Jose Cordero ${ }^{1}$, Hongbin Deng ${ }^{1,2}$ and Yantao Shen ${ }^{1 *}$
}

\begin{abstract}
The research work aims at developing a real-time non-invasive metabolism and blood circulation surveillance system for monitoring human's health condition by sensing the various bio-signals on the human body. Our goal is to use the developed system to study the functions and characters of organs and tissues that highly relate with the metabolism and blood circulation system, and also, it is expected to help modeling the entire circulation system. At phase I of the research, in this paper we focus on developing a new low-cost, portable, high-accuracy, non-invasive radial pulse sensor. Inspired by touch capability and related biomechanical advantage of human fingertip, the mechanical design of the sensor mimics the physiological structure of human fingertip. The biomimetic sensor is then well calibrated using a high-accuracy force sensor, and the model is accurately identified by the system identification method. Further the calibrated sensor is applied to diagnose the arterial stiffness by measuring the augmentation index (Al) which is the important biomarker of vascular aging. Preliminary results demonstrate the sensor performance that it is capable of non-invasively, accurately, and reliably measuring radial pulse signals at real time, as well as to quantitatively determine the vessel aging.
\end{abstract}

Keywords: Biomimetic; Non-invasive; Radial pulse sensor

\section{Background}

Cardiovascular system is built by central and systemic circulation systems, which generates the blood flow by the heart and transmits the blood to the whole body through the vessel pathway [1]. Associated with the blood flow, the pulse is an important signal containing the pathological information like heart function, blood flow resistance, vessel wall elastic, and blood viscosity. These pathological changes are usually expressed on strength, frequency, altitude, and waveform variation of the pulse signal. Instead of invasive thermodilution methods and complicated noninvasive impedance-cardiography, the pulse wave analysis can thus be an alternative way to monitor the cardiovascular system.

One of these pulses is the radial pulse. A diagnosis method relying on the radial pulse signals detected by

\footnotetext{
*Correspondence: ytshen@unr.edu

1 Department of Electrical and Biomedical Engineering, University of Nevada at Reno, 1664 N Virginia Street, Reno, NV 89557, USA

Full list of author information is available at the end of the article
}

physician fingers can be traced back to $500 \mathrm{BC}$ in China [2]. The first mechanical device can measure the arterial pressure waveform at radial called sphygmograph which was developed and improved in the nineteenth century [3]. Later, more and more researchers studied into this area, and different kinds of devices to measure the radial pulse wave were investigated. Nowadays, there are various commercial pressure waveform measurement devices developed by different companies and groups. These devices generally use technologies like RF-signal of an ultrasound, applanation tonometry, photoplethysmography, piezoelectric sensor, oscillometric, mechanotransducer, and volume plethysmography [4]. However, most of these sensing devices are only capable of measuring the systolic blood pressure, diastolic blood pressure, and heart rate [5]. The typical calibration for them is only a static process that relies on several discrete reference values from the cuff-based blood pressure sensors $[5,6]$. This results in most of them could not achieve radial pulse details and dynamic behaviors that contain rich

\section{Springer}

C 2014 Luo et al: licensee Springer. This is an Open Access article distributed under the terms of the Creative Commons Attribution License (http://creativecommons.org/licenses/by/4.0), which permits unrestricted use, distribution, and reproduction in any medium, provided the original work is properly credited. 
physiological and pathological information reflecting cardiovascular disease and vessel aging. In addition, most of devices are not cost effective and are unwearable, and this limits them to be extensively used for continuously monitoring the pulse signals of users. Without continuously monitoring the signals, the history of health condition related to cardiovascular diseases cannot be recovered for helping the diagnosis process and certain important physiological and pathological characteristics may be generally missed. For example, some characteristics from the pulses are significant during the intense exercise or the sleeping time due to the heavy load of the cardiovascular system and increasing of the blood viscosity; however, they may be lost without wearing a portable pulse sensor that has continuous monitoring capability. Recently, some research work also mentioned that continuous monitoring of the pulses will help to prevent the varied cardiovascular diseases at the early stage as well as to alarm users when the pulse signals become singular so as to avoid the situations like stroke, myocardial infarction, and sudden death $[2,7]$. In prospect of the increase in demand, developing a low-cost, wearable pulse sensor that can offer real-time continuous monitoring, immunizes mechanical and electronic noises, as well as displays some basic diagnostic results is becoming one of the most popular research topics in this area $[5,6]$.

This paper aims at developing a real-time non-invasive metabolism and blood circulation surveillance system for monitoring human's health condition by sensing the various bio-signals on the human body; as its phase 1 , we firstly address developing a high-accuracy, highsensitivity, low-cost, non-invasive, and wearable radial pulse sensor for the system. More importantly, inspired by touch capability and associated biomechanical advantage of human fingertip, the mechanical design of the sensor mimics is the human fingertip physiological structure. In addition, to pursue the accuracy, the biomimetic sensor is well calibrated using high-accuracy force sensor and the model is accurately identified by the system identification method. The extended applications of the sensor include monitoring human's health condition in real time to support medical diagnosis process and physiological and pathological studies. It can be also expected to prevent and alarm the varied cardiovascular diseases at the early stage and boosts related clinical studies. Extensive calibration results show the good performance such as high reliability, high accuracy, and high sensitivity of the developed biomimetic sensor. In the first application, the sensor was successfully used to measure one critical factor called augmentation index (AI) that is related to arterial stiffness and cardiovascular diseases [7].

The paper is structured as follows. In Section 'Methods', Subsection 'Biomimetic radial pulse sensor design' reviews the human fingertip physiological structure and describes the mechanical and electrical designs of the biomimetic radial pulse sensor. Subsection 'Sensor calibration, identification, and verification' presents the calibration and identification processes of the developed sensor and validates the biomimetic performance of the sensing structure. Section 'Results and discussion' depicts the radial pulse measurement performance of the sensor, the clinical application and its preliminary results. Finally, Section 'Conclusions' concludes the work.

\section{Methods}

\section{Biomimetic radial pulse sensor design}

\section{Human fingertip physiological structure}

Finger palpation methods have been performed many centuries, and they are still widely applied in the clinic process. The mechanism of the palpation is that the physical properties containing the physiological and pathological information of the object that is contacted with the fingertip can be purely transferred to mechanoreceptors inside of the fingertip through its fine physiological structure with mechanical filtering functions [8]. This indicates that human fingertip owns the biomechanical advantages for achieving high-performance touch/force sensing and inspires us to mimic it for designing our radial pulse sensing structure. To start the mimicking, we firstly review the physiological structure of the human fingertip. Figure 1 shows the anatomy of the fingertip and nail, and there are several layers for the whole fingertip structure constituted with different organisms. The epidermis is the first layer from the bottom to the top, which works as a protective layer isolating the interior of the fingertip from outside. Dermis is the interlayer next to epidermis and is distributed with various types of mechanoreceptors that can sense touch, pressure, and vibrations from 0.5 to

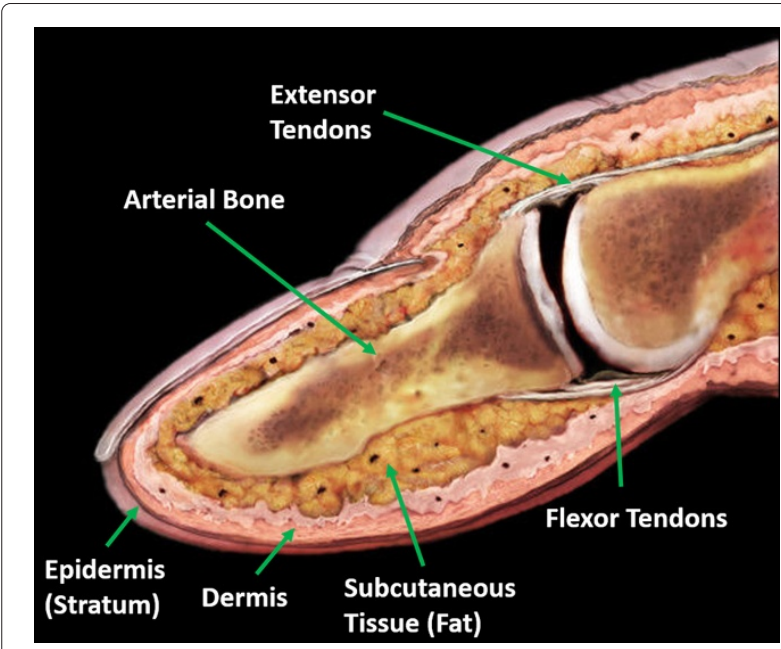

Figure 1 Illustration of human fingertip and nail anatomy. Adapted from [11]. 
$500 \mathrm{~Hz}$ [8]. Subcutaneous tissue is mainly a fat layer with $60 \%$ to $72 \%$ fluid in volume [9] and works like a damper to remove the unwanted information to the mechanoreceptors. The bone controlled by extensor and flexor tendons can move up and down to provide both passive and initiative movements for the effective touch and supports the fingertip as well. Due to the complex mechanical behavior and structures of the skin and subcutaneous, two simplified biomechanical models of the fingertip were reported [10]. These models include mechanical modeling of combined bio-components such as the bone, fat, soft tissues, and skin membrane. The results validating biomechanical advantages inspire us to design a radial pulse sensor that mimics the physiological structure of the human fingertip. As shown in Figure 1, several bio-components are arrowed and they are mimicked in designing our sensor components as shown in Figure $2 \mathrm{~b}$.

\section{Mechanical design of biomimetic sensor}

Figure 2 illustrates the mechanical design of the biomimetic radial pulse sensor using SolidWorks. Figure 2a displays the three-view diagram of the sensor structure. The length of the sensor is $50 \mathrm{~mm}$, the width is $30 \mathrm{~mm}$, and the height is $25 \mathrm{~mm}$ (without the adjustable screw); two grooves at two sides of the sensor can attach an adjustable belt to help wear it at the radial, or other body locations such as brachial, carotid, popliteal, and even superficial temporal comfortably. Figure $2 b$ details the sensor components that functionally mimic the fingertip bio-components and their functions. All components except the sensing film and polydimethylsiloxane (PDMS) damper were made by the 3D printer using the solid plastics. The diameter of the skin contact ball is $10 \mathrm{~mm}$, back side of the ball is glued with the sensing film, and the open side directly contacts with the radial

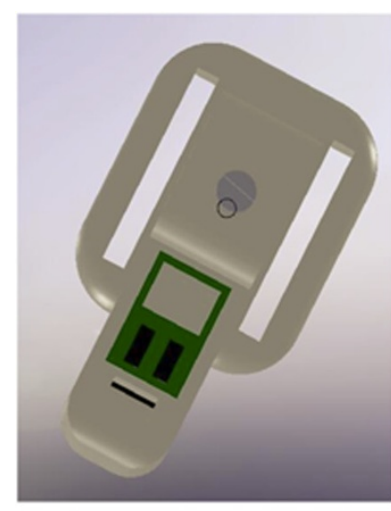

a

Top View

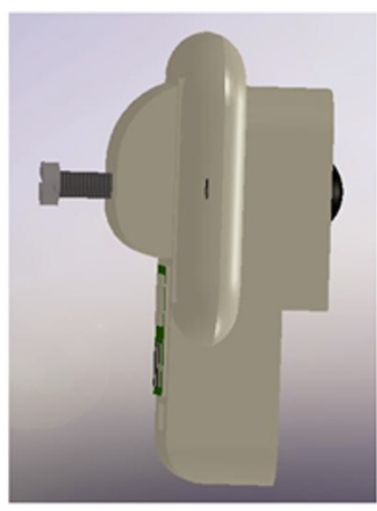

Side View

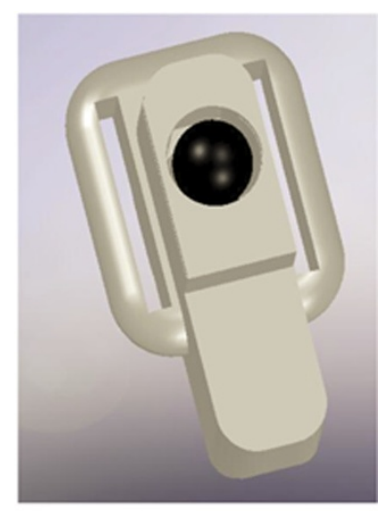

Bottom View

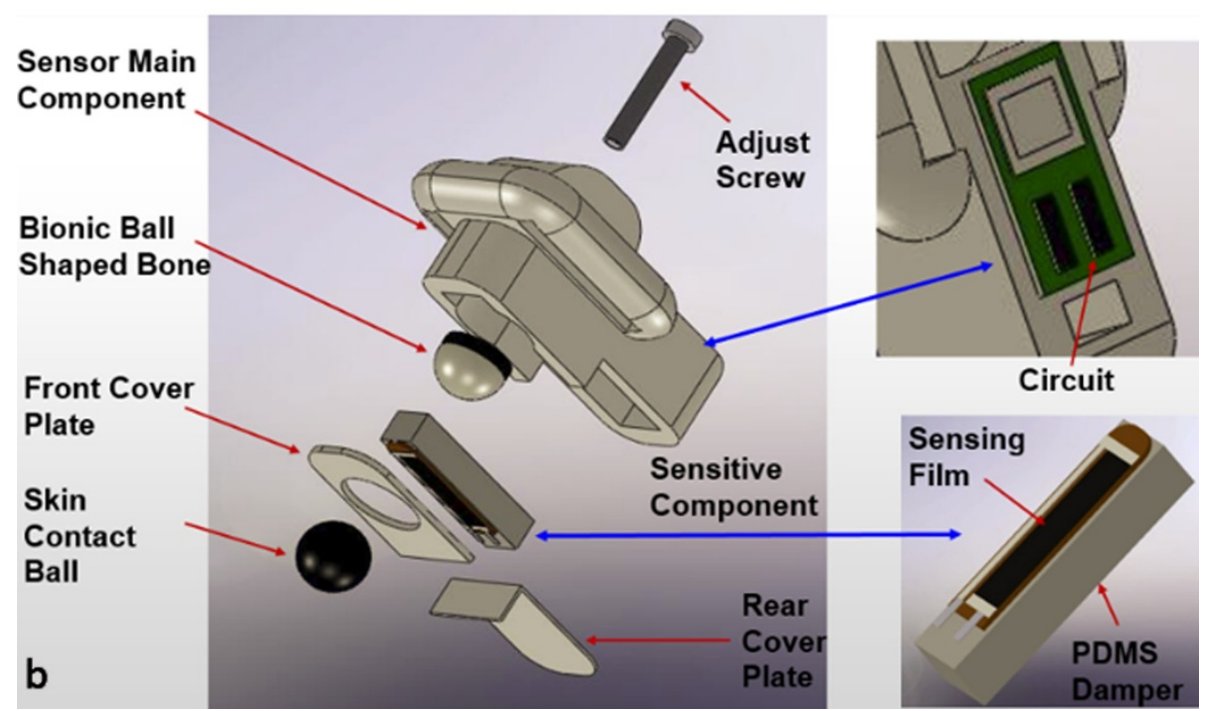

Figure 2 Illustration of mechanical design of the biomimetic radial pulse on SolidWorks. (a) Three-view diagram of the radial pulse sensor and (b) 3D assembly diagrams and components of the pulse sensor. 
pulse location of wrist. The rigidity of the ball is carefully chosen so that it can transfer the radial pulse signal to the sensing film without significant mechanical loss. The rigid contact ball design can also help user to quickly find the exact pulse points. The sensing film is one of the key components that mimic the mechanoreceptors of fingertip for the developed pulse sensor. It is an ultrasensitive hybrid carbon/polymer-based piezoresistive (HCP) film with thickness of $0.127 \mathrm{~mm}$, width of $3 \mathrm{~mm}$, and length of $12 \mathrm{~mm}$. The film has high sensitivity, very low thermal drift, and low hysteresis that were reported in our previous study [12]. The sensing film is glued on the elastic PDMS damper, the film will be deformed, and its resistance will change following the strength of transferred radial pulse signals .

The elastic PDMS damper is another key component in the sensor. It mimics subcutaneous soft tissues and fat in the human fingertip. To reach or close to the function of the soft tissues and fat, the hardness of the damper is an important factor. In this work, a digital shore hardness tester (TYPE A, range: 0 to $100 \mathrm{HA}$, resolution: $0.5 \mathrm{HA}$, accuracy: $\leq \pm 1 \% \mathrm{HA}$ ) was firstly used to estimate the hardness of the human fingertips. The average hardness of the fingertips measured by the digital shore hardness tester is around $30 \mathrm{HA}$. To make an elastic damper with the similar hardness, Sylgard 184 Silicone Elastomer (Dow Corning Corp., Midland, MI, USA) (temperature range $-45^{\circ} \mathrm{C}$ to $200^{\circ} \mathrm{C}$ ) was selected. Five different samples with different mixing ratios between base liquid (part A) and curing agent (part B) were tested in order to achieve the hardness that is close to the hardness of the soft fingertip. Vacuumed Thermo Scientific Nalgene Chambers (Thermo Fisher Scientific, Waltham, MA, USA) was then used to store all the mixed silicone elastomer liquids for 48-hour curing process at the room temperature. After cured, five different hardness PDMS elastic dampers were made using different mixing ratios between base liquid and curing agent such as 1:2, 2:3, 1:1, 3:2, and 2:1. Through a long-time aging test, we found that all hardness of all damper samples gradually reaches the constant values after 33 days, as illustrated in Figure 3a. Especially for the sample with mixing ratio 1:2 (base liquid 1: curing agent 2), it can be found in Figure 3b, its hardness was measured around $30 \mathrm{HA}$ that is the most close to the average hardness of the human fingertips. This PDMS sample was then chosen to be an elastic damper for the biomimetic sensor. In our design, the damper is a cuboid with a thickness of $3 \mathrm{~mm}$ (matches the thickness between the skin and the bone of fingertip), width of $3 \mathrm{~mm}$, and length of $15 \mathrm{~mm}$. In addition, the ball-shaped bone in the sensor mimics the arterial bone in the fingertip. The adjustable screw serves as the regulating unit of the bone. It functions as the extensor and flexor tendons in the fingertip. As shown in Figure 2b, all centers of the screw, bionic ball-shaped bone, and skin contact ball are aligned on a line in order to reduce the shear force and twist distortion during the operation.

\section{Electronics design}

The electronic circuit interfacing with the HCP sensing film was designed and custom-built for the sensor. It is used to process and to transform the signals that are from the film resistance changes caused by the radial pulse deformation. Since a preload force is applied on the sensing film when the sensor is worn at the radial, the gain and sensitivity of the circuit needs to be carefully adjusted to guarantee high sensitivity and high accuracy of the sensor, as well as to avoid saturation of the measured signals. To meet the requirement, as shown in Figure 4, a custombuilt Wheatstone quarter bridge circuit with an AD620 amplifier is employed.

In the schematic, $R_{6}$ is the resistor that represents the value of the resistive layer on the HCP sensing film. Its resistance is changed following the micro deformation caused by the radial pulse. At the top of $R_{6}$, a potentiometer is used to trim the bridge circuit through removing the zero offset. $R_{1}, R_{2}$, and $R_{5}$ are the constant resistors with the same resistance as the undeformed resistive layer. $R_{3}$ is the resistor used to modify the gain of the amplifier AD620, and the maximum gain of the amplifier can reach 10,000 . The circuit output $V_{o}$ is the analog voltage signal that is input to a PC for further display and processing through a 16-bit and $200 \mathrm{kS} / \mathrm{s}$ DAQ system PCI-DAS6013 made by Measurement Computing Corp.

\section{Sensor calibration, identification, and verification Sensor calibration setup}

As shown in Figure 5, all calibration and verification processes were implemented on a high-performance Newport vibration-isolated workstation. The calibration and verification procedures were automatically performed by the computer program in order to minimize the environmental noises and human effects.

Two high-performance sensors, a Shimpo FGV-1XY digital force gauge (Shimpo Instruments, Itasca, IL, USA) (capacity: $5 \mathrm{~N}$, resolution: $1 \mathrm{mN}$, high accuracy: $\pm 0.2 \% \mathrm{FS}$ ) and a Baumer OADM 20I6X41/S14F laser distance sensor (Baumer Electric, Southington, CT, USA) (range: $70 \mathrm{~mm}$, resolution: $20 \mu \mathrm{m}$ ), were employed in the calibration process. The laser distance sensor OADM 20I6X41/S14F was set up on the vibration-isolated workstation and its laser beam perpendicularly shoot on the moving stage of the micromanipulator for tracking the displacement change of the skin contact ball in the developed radial pulse sensor, when the digital force gauge is controlled to push/release the contact ball during the calibration. The pushing/releasing process was controlled by a Sutter MPC 385 3-D micromanipulator (Sutter Instruments, Novato, 

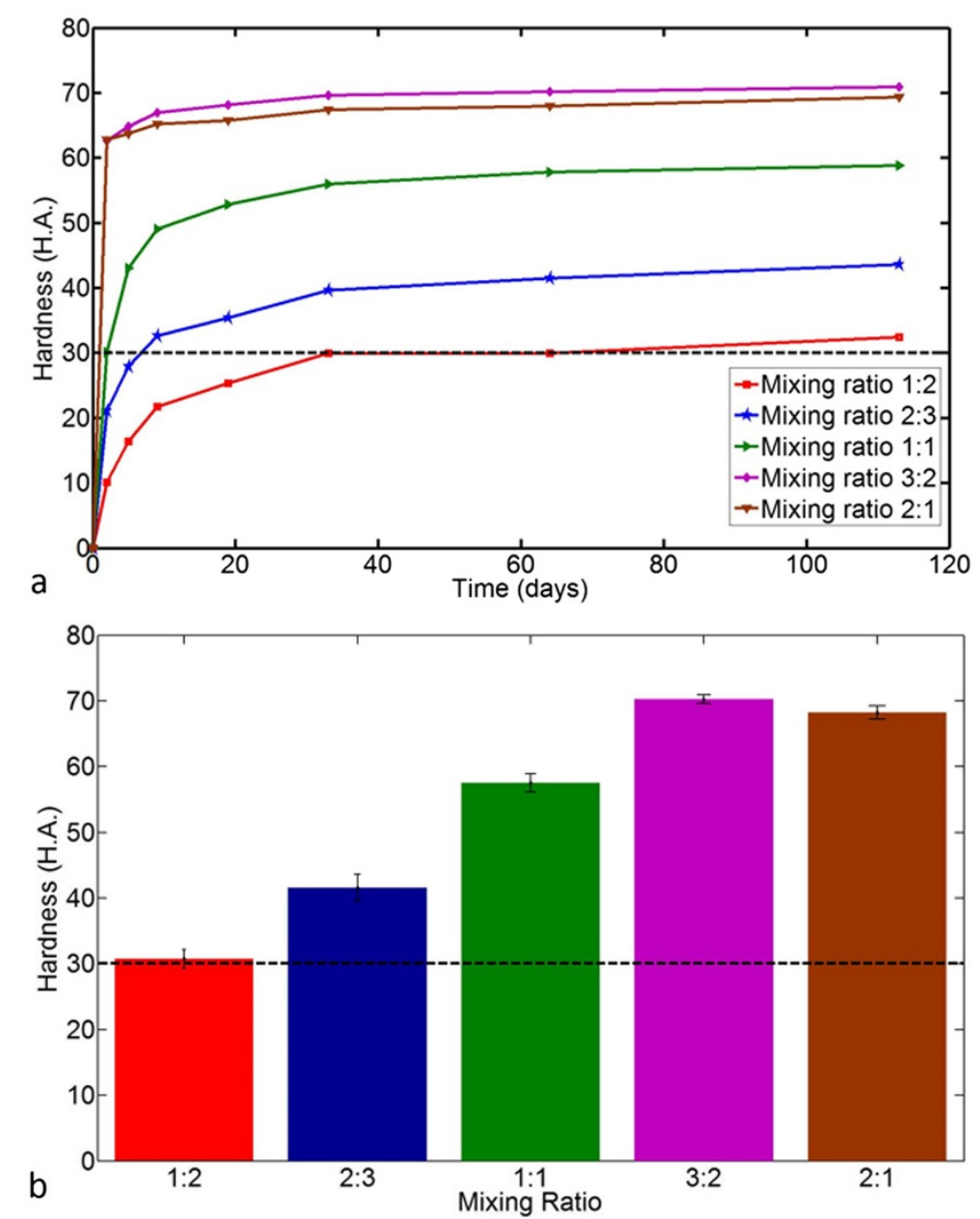

Figure 3 Long-time aging test result of multiple PDMS samples. (a) Five different hardness PDMS elastic dampers made by five different mixing ratios. (b) The damper made by the mixing ratio 1:2 has the hardness of 30 (after 33-day curing process) that is close to the average hardness of human fingertips.

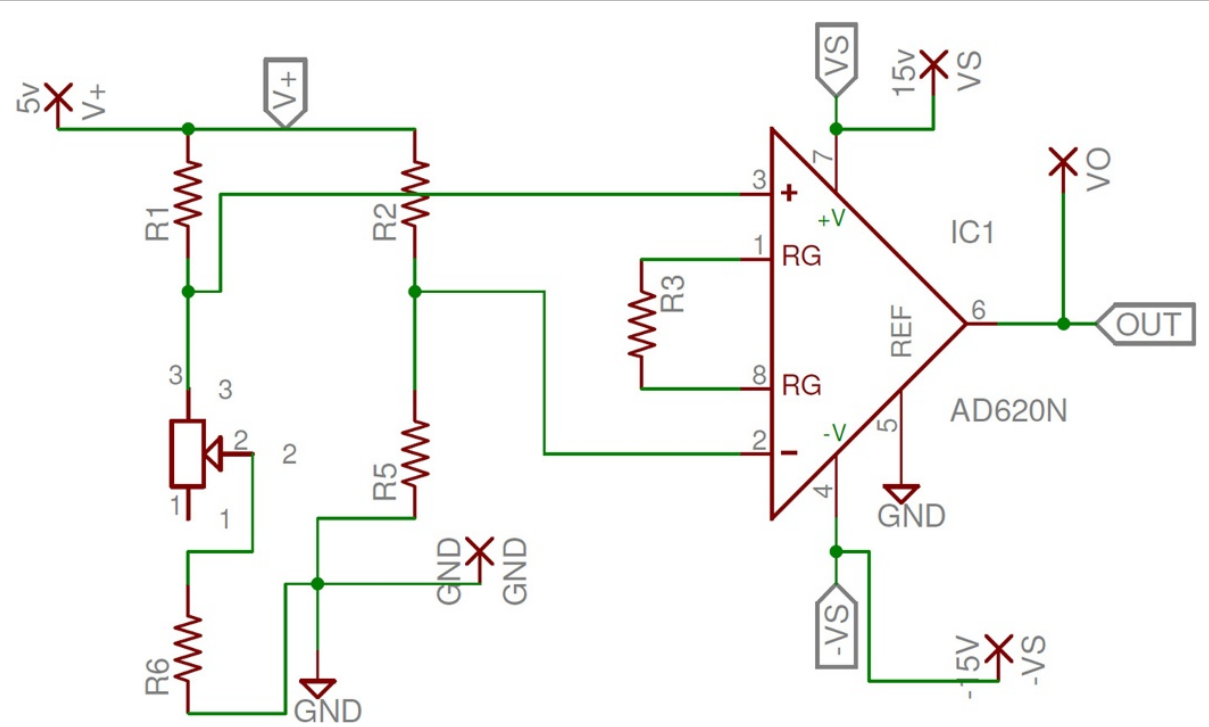

Figure 4 Circuit schematic of the pulse sensor. 


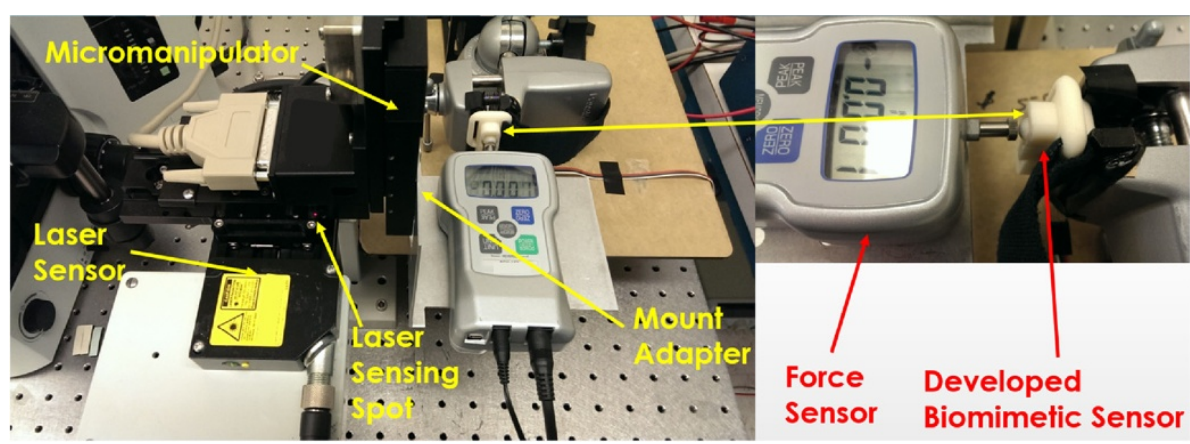

Figure 5 The setup for calibrating the biomimetic radial pulse sensor.

CA, USA) (range: 25,000 $\mu \mathrm{m}$, resolution: $1 \mu \mathrm{m}$ ) where the digital force gauge is attached on the micromanipulator. The process can generate $150-\mu \mathrm{m}$ height square wave pushing/releasing movement by the program-controlled micromanipulator. Meanwhile, the force information was recorded by the force gauge. In the calibration process, the developed pulse sensor was fixed on the vibration-isolated workstation through a clamping stander. Note that, to meet the real condition that the preload force is applied to the developed sensor during wearing it, an average 1.6 $\mathrm{N}$ preload force to the contact ball was initially set by the force gauge FGV-1XY before and during the calibration. In addition, the designed electronic circuit with the gain of 50 times was interfaced with the developed sensor. All signals were recorded to a PC through the DAQ board PCI-DAS6013 (Measurement Computing Corporation, Norton, MA, USA) (resolution: 16 bits, range: \pm $10 \mathrm{~V}$, number of channels: 16 , accuracy: $\pm 8.984 \mathrm{mV}$, speed: $200 \mathrm{kS} / \mathrm{s}$ ) with sampling rate of $1 \mathrm{kS} / \mathrm{s}$.

\section{Identification and calibration}

Figure 6 plots all experimental data including pushing/releasing force, the developed sensor output, and pushing/releasing displacement on the skin contact ball of the sensor. The first row is the force curve measured by the digital force gauge FGV-1XY. The second row plots the voltage output from our developed pulse sensor, and the last row shows the displacement detected by the laser distance sensor OADM 20I6X41/S14F. The data demonstrates the developed sensor which has the fast response speed and good dynamic behavior.

Based on the experimental data shown above, the transfer function model between the developed pulse sensor voltage output and the force measurement by the force gauge FGV-1XY can be found through the system identification method developed in MATLAB. Here, the sensor output is used as the input, and the force measurement is used as the output (reference). The Laplace

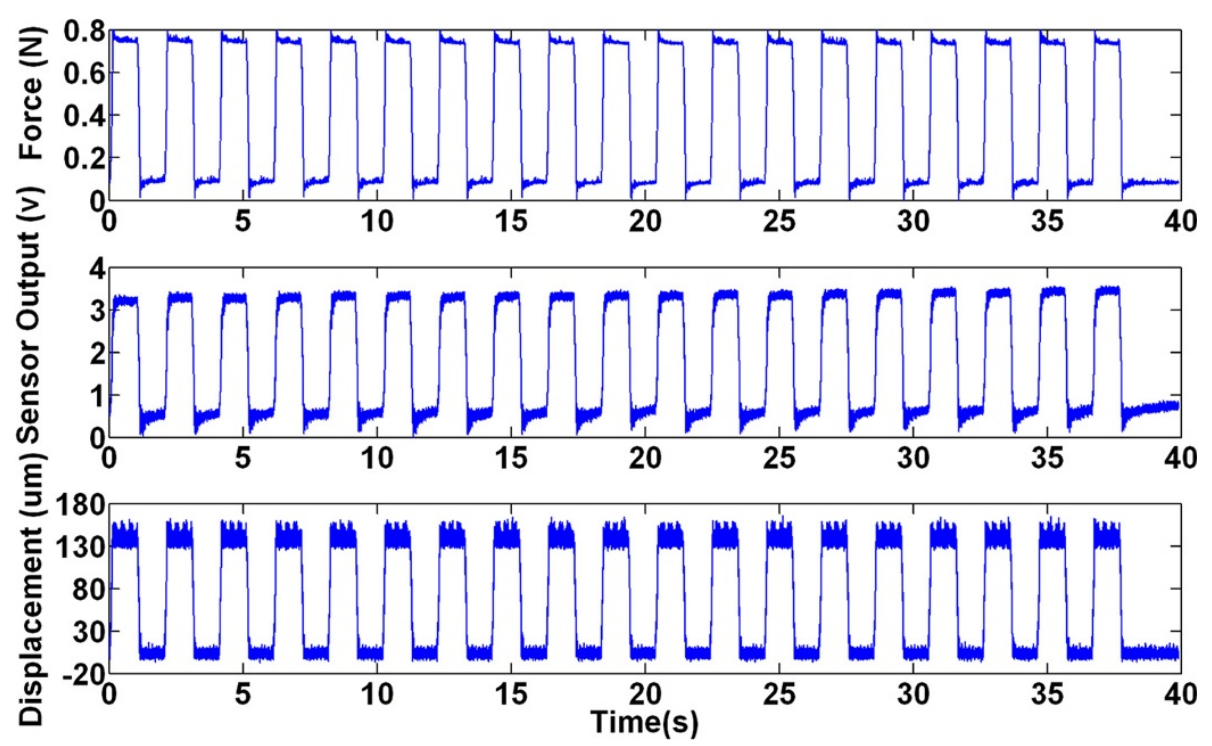

Figure 6 Raw data of force, the pulse sensor output, and displacement. 


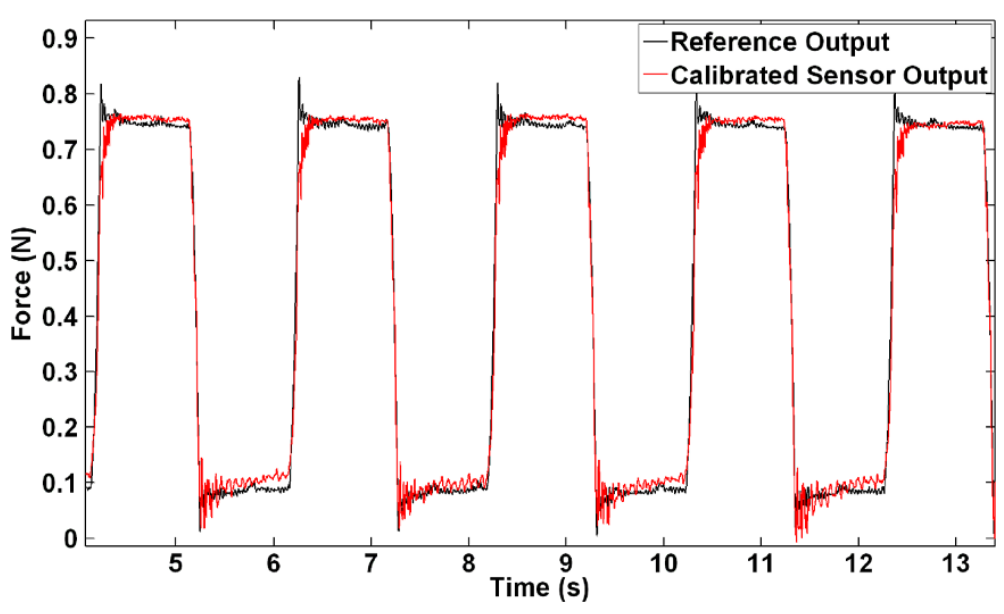

Figure 7 The calibrated sensor output and the reference measurement by force gauge FGV-1XY.

transfer function is identified as shown in the following equation:

$$
G(s)=K \frac{1+T_{z} s}{\left(1+T_{p 1} s\right)\left(1+T_{p 2} s\right)\left(1+T_{p 3} s\right)}
$$

where the gain $K=0.20998$, the coefficients $T_{p 1}=0.001$, $T_{p 2}=9.7751, T_{p 3}=0.0023316$, and $T_{z}=11.063$. By applying the identified model in Equation 1 to the raw sensor voltage output, as shown in Figure 7 , we can see that the fitting rate between the reference force measurement (black line) and the calibrated sensor force output (red line) is $90.75 \%$ high and the maximum error at peaks is $3.25 \%$.

Further, Figure 8 plots the comparison results on frequency-amplitude relationship between the calibrated sensor output signals and the reference signals. The consistence between the two results further indicates the calibration performance of the sensor, that is, the sensor is well calibrated and can be used for the radial pulse measurement.

\section{Biomimetic performance verification of sensing structure}

The calibration results of the sensor have demonstrated the good mechanical to electronic conversion performance of the developed sensor. To further verify the biomimetic performance of the sensing structure that is close to the structure advantage of the human fingertip, an experiment was conducted to investigate the force-displacement relationship of serially connected components in the sensing structure consisting of a rigid skin contact ball, a sensing film, a silicone elastic damper, and a ball-shaped bone structure. In this experiment, the setup is similar to the calibration one described in Subsection 'Sensor calibration, identification, and verification'. Instead of using 150- $\mu \mathrm{m}$ height square wave motion, $150-\mu \mathrm{m}$ height triangle wave movement was generated by the computer program to push

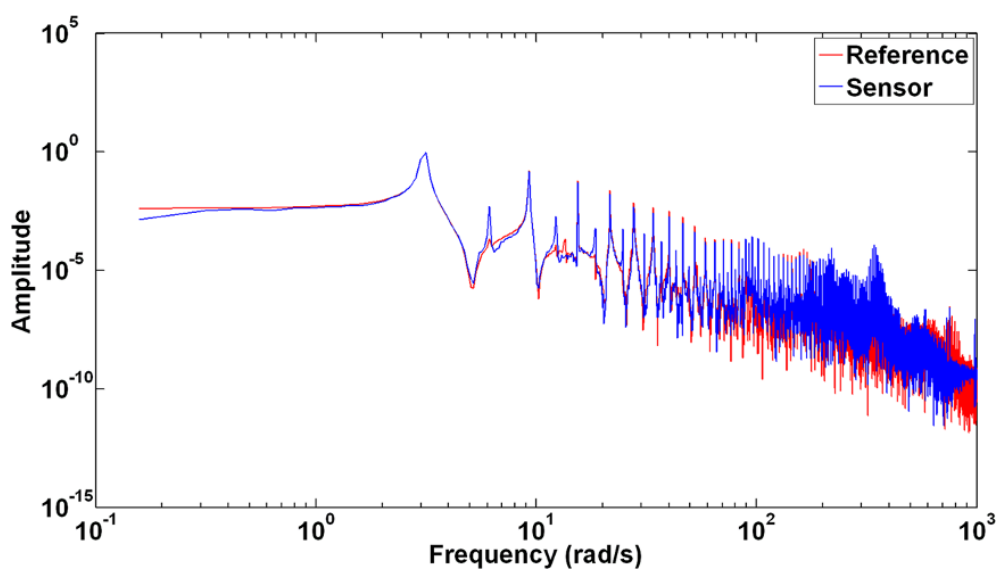

Figure 8 Comparison on spectral analysis results between the calibrated sensor output and reference signals. 


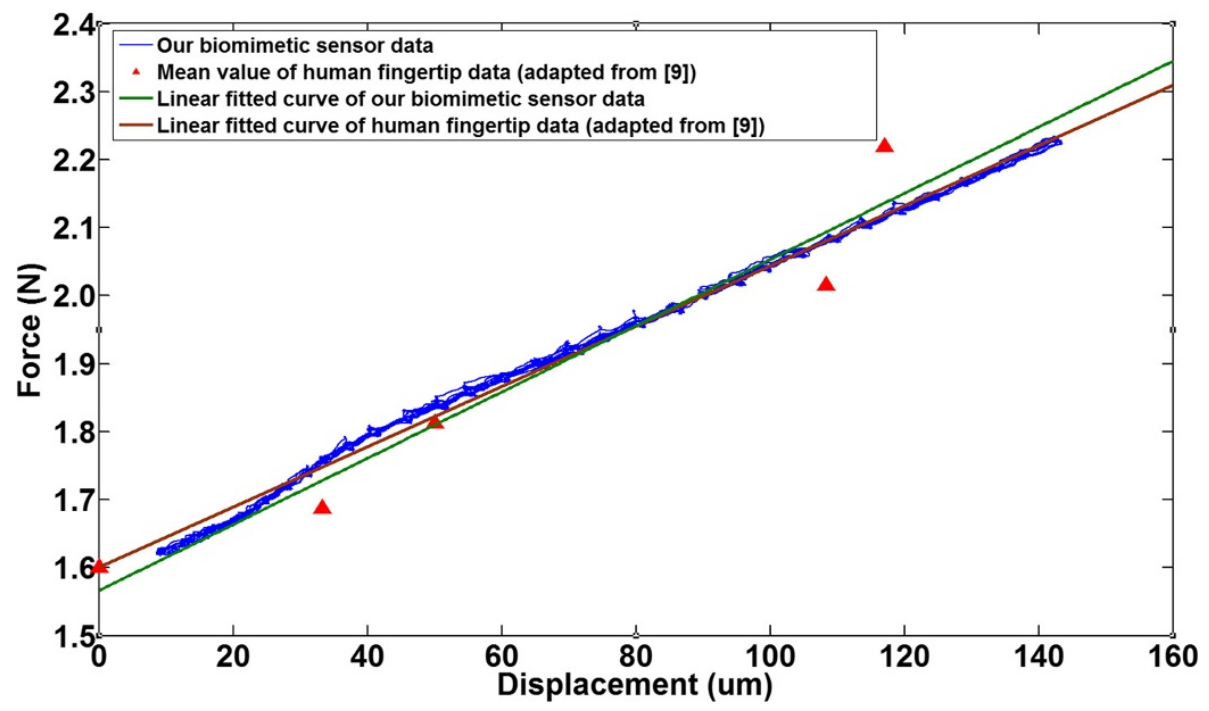

Figure 9 Relationship/comparison between force and deformation of both the sensing structure and human fingertip structure.

and release the sensing structure through the contact ball. The laser distance sensor OADM 20I6X41/S14F was set to a higher resolution but with a short range (range: $30 \mathrm{~mm}$, resolution: $4 \mu \mathrm{m}$ ) to accurately detect displacement/deformation of the sensing structure caused by pushing and releasing operations.

Figure 9 plots the experimental force-displacement data points of the sensing structure, the mean value data points from real human fingertips conducted by Serina et al. in [9], and two curves fitted using the above two data point groups. Note that, a preload force of $1.6 \mathrm{~N}$ was applied to the sensing structure before conducting experiments. Some small spines in our experimental forcedisplacement data curves were induced by the movement of the step driven motors of the micromanipulator. It is clear that our sensing structure force-displacement data points demonstrate a good linear relationship between the force and the displacement in several pushing and releasing experimental loops. The small overlap between these data points (curves) in several experimental loops indicates a very low hysteresis effect of the designed sensing structure. More importantly, our experimental result agrees well with the force-displacement behavior of the human fingertips conducted by Serina et al. [9]. In the figure, the brown linear fitted curve from the data points of our biomimetic sensor is very close to the green linear fitted curve from the human fingertip data measured by Serina et al. [9] and it experimentally verifies the successful biomimetic design of our sensor.

\section{Results and discussion}

\section{Radial pulse measurement and results}

After the calibration process, the sensor can be used to measure the radial pulses. As shown in Figure 10, one user worn the developed biomimetic pulse sensor and placed his arm on the flat table where the user sits in front of. The developed acquisition and signal processing program in a PC is started, and it records the processed radial pulse signals from the sensor and the circuit and displays the results on the screen in real time.

Figure 11 shows the raw radial pulse signal (in red) and the calibrated radial pulse signal (in blue) of the user.
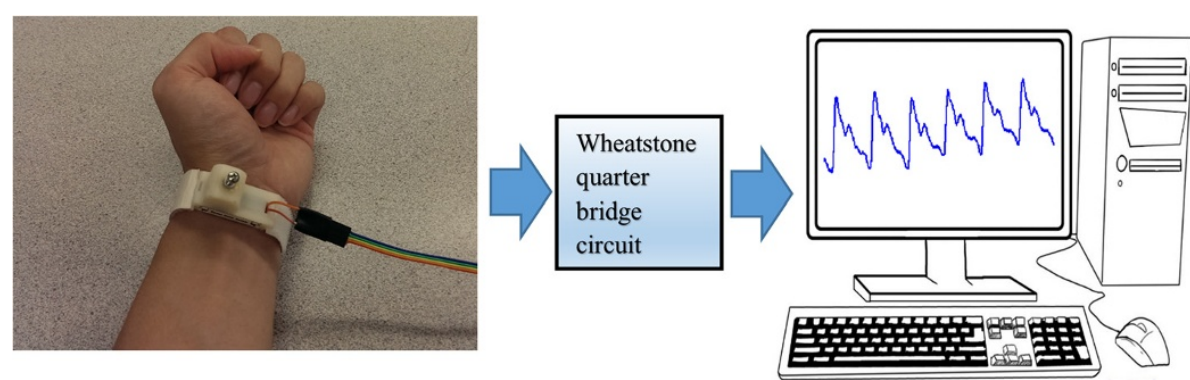

Figure 10 Illustration of the radial pulse measurement setup. 


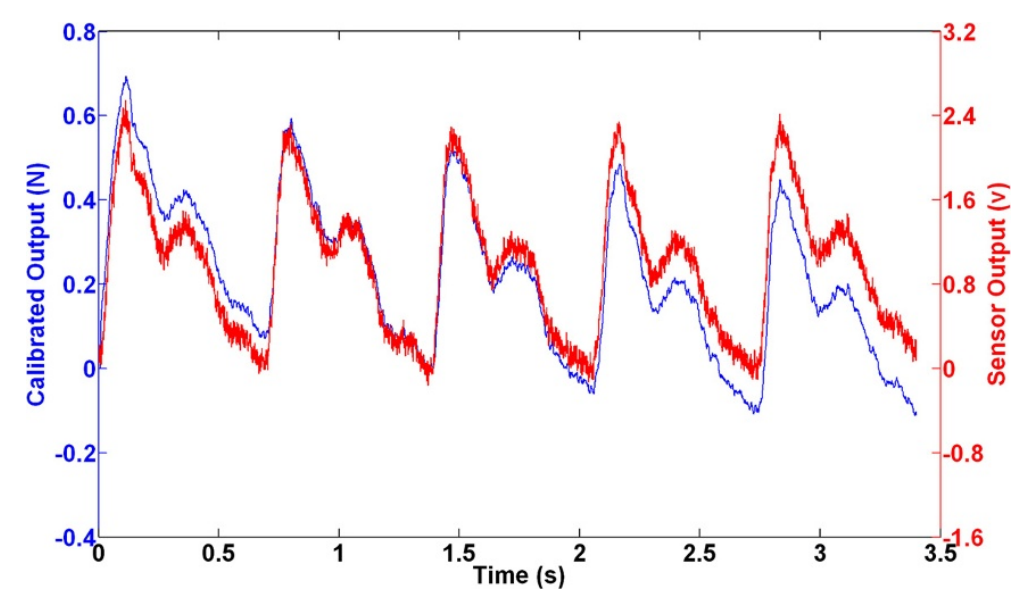

Figure 11 Radial pulse signal measurement and calibration results.

The results demonstrate the good measurement performance of the developed sensor as its raw data is very close to the calibrated data, as well as the detail of the pulse information is well revealed.

\section{Clinic application and results}

Next to the achieved accurate radial pulse signals using the sensor, in this section, one clinical application based on the radial pulse measurement is extensionally presented. The application is related to determining the arterial stiffness by computing the AI using the measured radial pulse signals of the sensor.

In the medical area, arterial stiffness is seen as an important biomarker of the cardiovascular risk [7]. The central augmentation index (cAI) is widely acknowledged as an evaluation factor for this biomarker. cAI is usually defined as the ratio of difference between the first and second systolic peaks with the pulse pressure. As shown in Figure 12, the complex pulse waveform (in black) at carotid (central) artery contains a forward wave (the first one is in blue) generated by the heart and a backward wave (in red) reflected from the peripheral artery. The central augmentation index is then defined as cAI = $(\mathrm{Pi}-\mathrm{PS}) /(\mathrm{PS}-\mathrm{PD})$ that presents the ratio difference at carotid (central). This index was first reported by Murgo et al. [13], and then, Kelly et al. [14] discovered the way to find the inflection point using the fourth derivative of the pulse waveform. Later, Takazawa et al. [15] researched the radial (peripheral) pulse augmentation index (rAI) and found that rAI is the ratio of the height of late systolic pressure to the early systolic pressure. They also demonstrated the automatic way to find the late systolic pressure point at radial artery by fourth derivative of the radial pulse waveform [15].

Recent research shows that the central augmentation index cAI can be estimated by applying a transfer function to the radial pulse augmentation index (rAI) $[16,17]$, or can be obtained directly from the radial pulses [18] due to

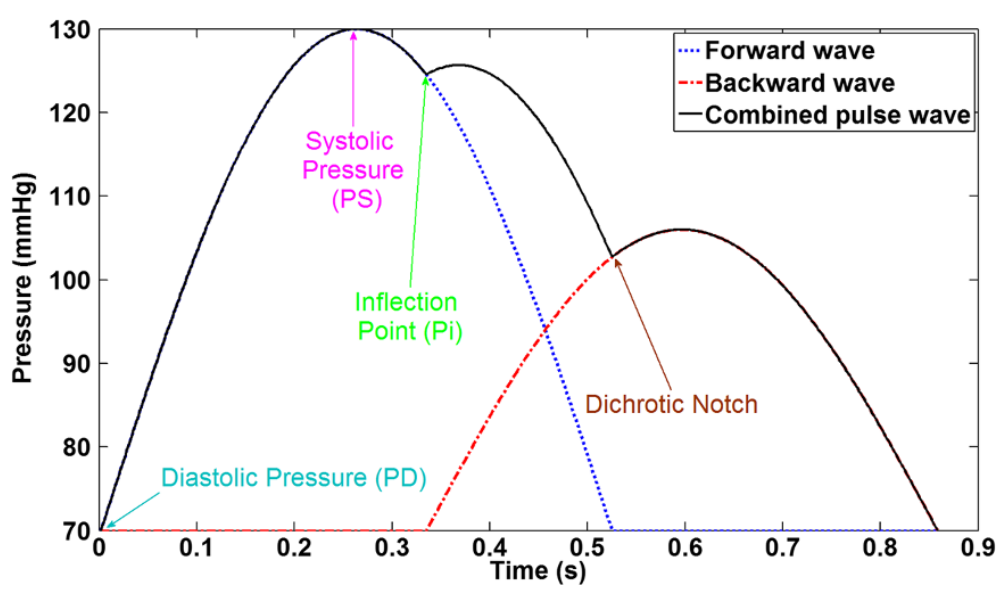

Figure 12 Illustration of C-type waveform at carotid artery. 


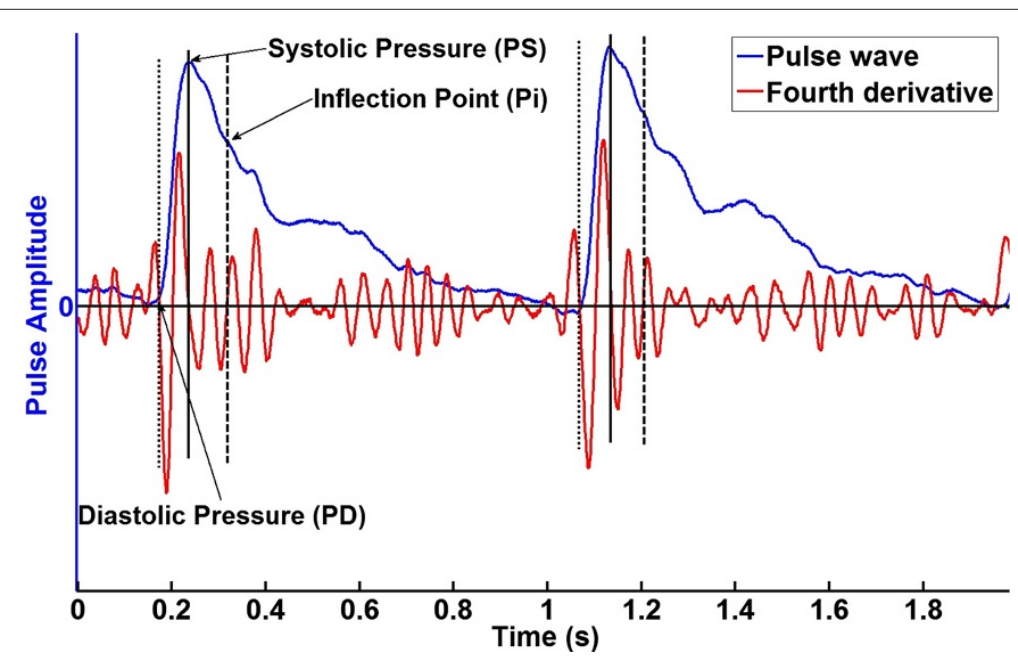

Figure 13 Measured radial pulse signal and its processing for achieving the augmentation index.

high correlation. This indicates $\mathrm{rAI}$ can be also used as the evaluation factor like cAI. In this paper, based on the radial pulse measurement using the developed sensor, we preliminarily start to investigate radial augmentation index rAI as well to correlate it into the cAI for determining the arterial stiffness.

As shown in Figure 13, the blue curve presents the pulse wave measured by our calibrated sensor and the red curve is the fourth derivative of the pulse wave after filtering using Savitzky-Golay FIR smoothing filter. In addition, the dot, solid, and dash vertical lines indicate the diastolic pressure (PD) point, systolic pressure (PS) point, and inflection point $(\mathrm{Pi})$, respectively. These points in time axis are determined when the fourth derivatives of the pulse wave cross to zero [15]. After determining these points, radial augmentation index, $\mathrm{rAI}=$
(Pi-PD)/(PS-PD) can be computed. The rAI is strongly correlated with the carotid (central) augmentation index (cAI), and the corrected results can be used to determined arterial stiffness and cardiovascular risk directly $[19,20]$.

To validate the augmentation index achieved by the pulse signals from the developed pulse sensor, a photoplethysmography (PPG) (Embedded Lab, HRM2511-E) sensor was synchronously employed for additional measurements during measuring the radial pulse. PPG signals can be used to compute the index of SI. The SI index is an equal factor as the augmentation index and can be used as an important value that evaluates the artery stiffness. The index is computed based on the height of the subject/user divided by the time delay between the subject's systolic and diastolic peaks [21], as illustrated in Figure 14.

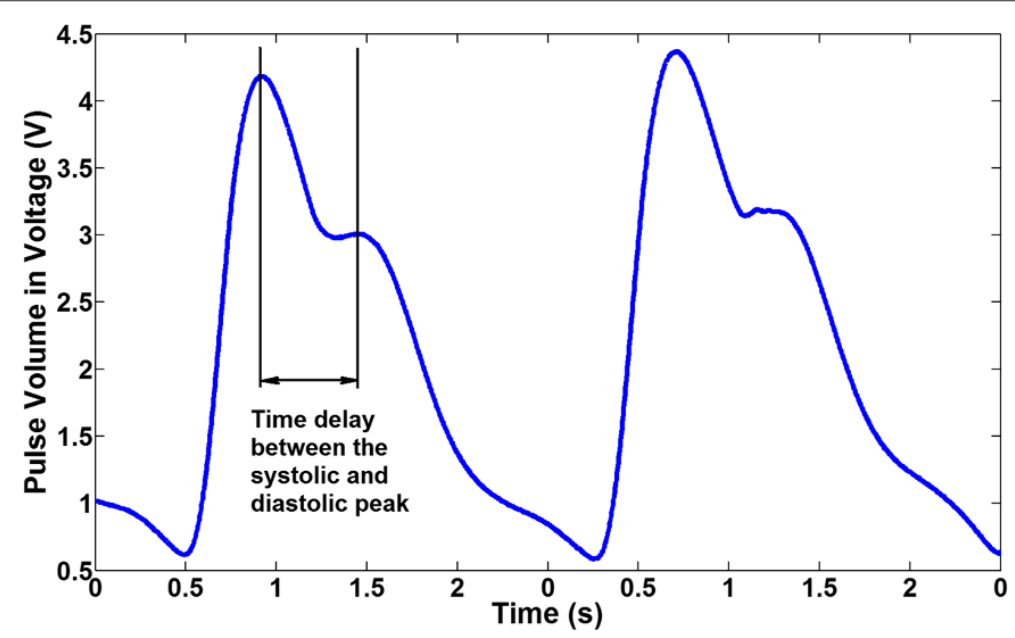

Figure 14 Measured PPG signals for achieving the SI index. 


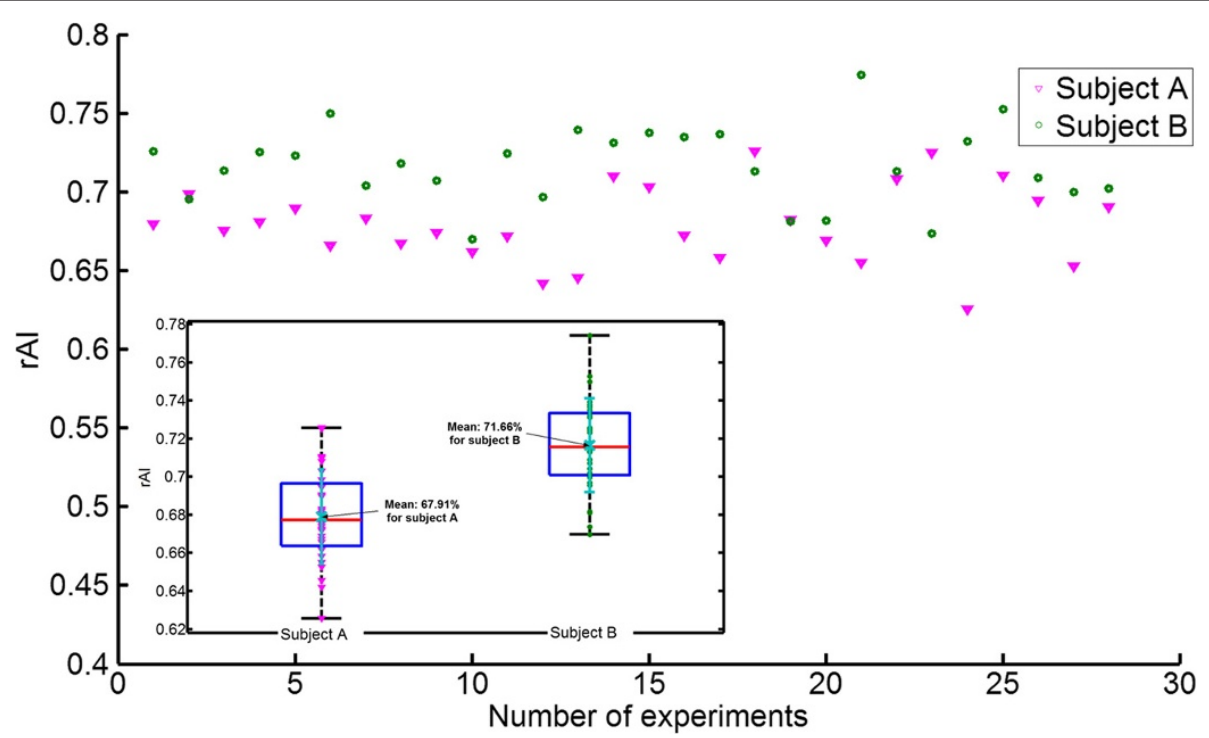

Figure 15 Experimental results and statistical analysis boxplot of two-subject's rAls (measured by our pulse sensor).

Note that, in this clinic test, two non-smoking health subjects without cardiovascular disease history, subjects $\mathrm{A}$ and $\mathrm{B}$, with the same height and similar weight were chosen to measure after 30 -min rest in a quiet room with more than 3-h fasted, also abstained from caffeine and alcohol. The developed pulse sensor was worn at the radial on the left arm of each subject. The PPG sensor was clamped at the index finger of the right hand of each subject. Both signals from our pulse sensor and the PPG sensor were collected by the DAQ board PCI-DAS6013 (sample rate: $1 \mathrm{kS} / \mathrm{s}$ ) in the same time.
Corresponding to Figure 13, Figure 15 shows twosubject experimental data of the rAI measured by our sensor and their statistical analysis results. Note that, experiments were conducted 28 times for each subject. In the figure, the pink triangle data points indicate the $\mathrm{rAI}$ values of subject $A$ and the green circle data points represent $\mathrm{rAI}$ values of subject $\mathrm{B}$. Embedded small figure at the left bottom of Figure 15 displays the statistical analysis results of two-subject experimental data points by the boxplot method with the mean and error bar. Both boxplot graphs represent the range between the 25th percentiles and 75th percentiles using the blue squares,

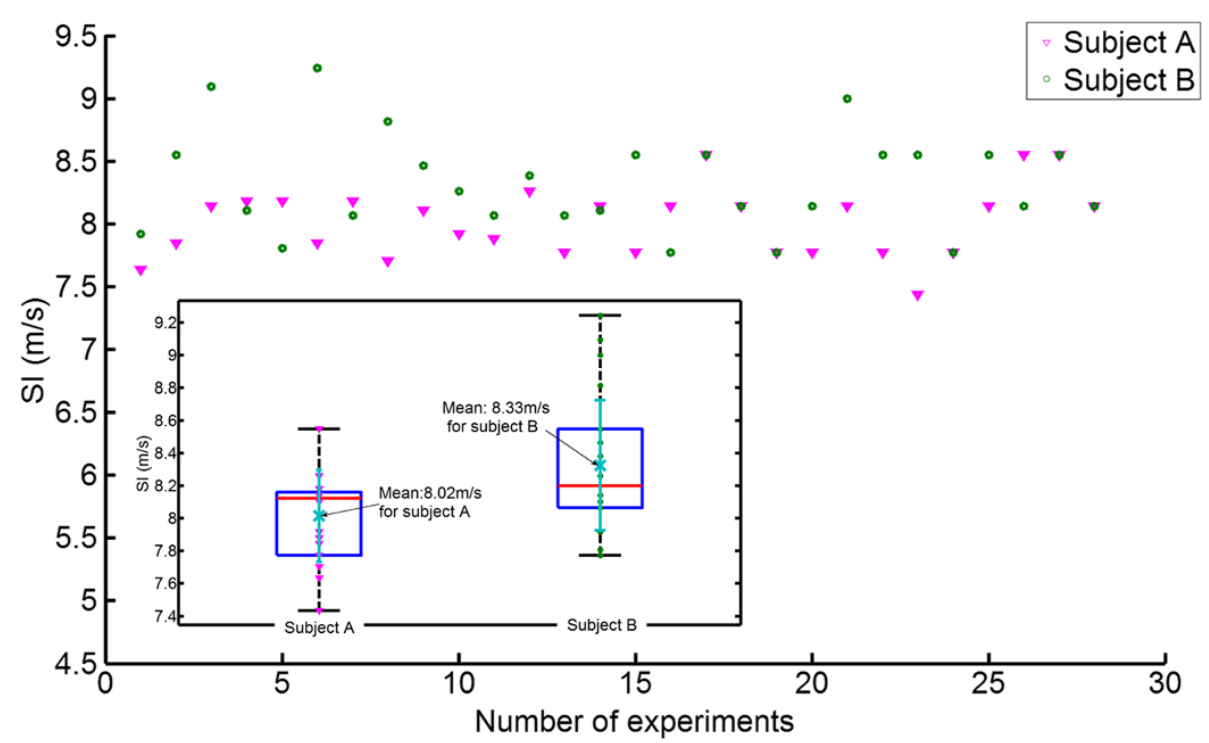

Figure 16 Experimental results and statistical analysis boxplot of two-subject's SIs (measured by the PPG sensor). 
Table 1 Physiological characteristics of the subjects and measured $\mathrm{Al}$ and $\mathrm{SI}$ indexes

\begin{tabular}{lcc}
\hline Characteristic & Subject A & Subject B \\
\hline Height & $1.71 \mathrm{~m}$ & $1.71 \mathrm{~m}$ \\
Weight & $73 \mathrm{~kg}$ & $75 \mathrm{~kg}$ \\
Body mass index & 25 & 25.6 \\
Age & 25 & 30 \\
Sex & Male & Male \\
Cardiovascular disease history & No & No \\
Al (our pulse sensor) & $67.91 \%$ & $71.66 \%$ \\
SI (PPG) & $8.02 \mathrm{~m} / \mathrm{s}$ & $8.33 \mathrm{~m} / \mathrm{s}$ \\
\hline
\end{tabular}

and the red lines in the blue squares are the medians of two data sets, respectively. The celeste blue cross and the vertical celeste blue line in the middle of each square show the mean and the standard deviation of the experimental data of each subject, respectively. Similarly, corresponding to Figure 14, 28 experimental results of two subjects on the SI index measured by the PPG sensor and their boxplot statistical analysis results are plotted in Figure 16. Both results in Figures 15 and 16 demonstrate that the vessel aging evaluated by both mean values of $\mathrm{rAI}$ and SI of subject $B$ is higher than the value of subject $A$. This result agrees well with age truth of two subjects and indicates our pulse sensor can achieve highly accurate and precision results for evaluating vessel aging that is highly related to subject age.

Table 1 summarizes all experimental tests. As shown in the table, the physiological characteristics of the two subjects are very close except for age. Two biomarkers, the mean value of $\mathrm{rAI}$ measured by the developed pulse sensor and the mean value of SI detected by the commercial PPG, which are related to the arterial stiffness, are listed. The AI index for the younger subject (subject A) is $67.91 \%$ while for the 30 years old subject (subject B) is $71.66 \%$ that is slightly stiffer than the value of the younger subject. In the normal condition, the augmentation index will increase as age increases $[14,22]$. The achieved AI results reasonably reflect the truth of arterial stiffness of the two subjects. In addition, the truth reflected by the measured AI index results is well validated by the SI index results from the PPG. These results clearly verify the sensor performance that the developed biomimetic sensor can be successfully used in determining the arterial stiffness and vessel aging and related clinic applications.

\section{Conclusions}

In this paper, inspired by touch capability and biomechanical advantage of human fingertip, we developed a highperformance radial pulse sensor with the highly sensitive, durable, low-cost HCP film, and biomimetic sensing structure. Calibration and verification prove the sensor performance that it could extract the accurate and reliable pulse signals non-invasively. One clinic application of the developed sensor is to determine the arterial stiffness based on the calculated augmentation index at radial. The results indicate that the developed sensor can be used in clinic applications and can be potentially extended to use in our real-time non-invasive metabolism and blood circulation surveillance system in the future.

\section{Competing interests}

The authors declare that they have no competing interests.

\section{Authors' contributions}

$Y L$ participated in all stages of the sensor design, carried out all calibration and experiments, performed data processing and analysis, and drafted the manuscript. SN and JC helped in conducting the experiments and data processing. HD participated in the design of the sensor. YS conceived the research, participated in the sensor design, data analysis and the research coordination, and revised the manuscript. All authors read and approved the final manuscript.

\section{Acknowledgements}

This research work is partially supported under the NSF Career Award CBET 1352006 and the Research Enhancement Grants (REG) from UNR office of the vice president for research and innovation.

\section{Author details}

${ }^{1}$ Department of Electrical and Biomedical Engineering, University of Nevada at Reno, 1664 N Virginia Street, Reno, NV 89557, USA. ${ }^{2}$ Department of Mechatronic Engineering, Beijing Institute of Technology, 5 South Zhongguancun Street, Haidian, Beijing 100081, China.

Received: 8 September 2014 Accepted: 24 October 2014

Published online: 18 November 2014

\section{References}

1. Green JF (1987) Mechanical concepts in cardiovascular and pulmonary physiology. 2nd edn. Philadelphia, Lea \& Febiger

2. Fan ZP, Zhang G, Liao S (2011) Chap. 2, Advanced biomedical engineering, Edited by Gargiulo GD. InTech, http://www.intechopen. com/books/advanced-biomedical-engineering

3. Kim DH, Braam B (2013) Assessment of arterial stiffness using applanation tonometry. Can J Physiol Pharmacol 91:999-1008

4. Avest ET, Stalenhoef AF, Graaf JD (2007) What is the role of non-invasive measurements of atherosclerosis in individual cardiovascular risk prediction? Clin Sci 112:507-516

5. Tu T, Chao PCP, Lee Y (2013) A new non-invasive cuff-less blood pressure sensor. In: Proceedings of the 2013 IEEE International Conference on Sensors, 857-860, Baltimore, MA, USA

6. Hsu YP, Young DJ (2013) Skin-surface-coupled personal health monitoring system. In: Proceedings of the 2013 IEEE International Conference on Sensors, 210-213, Baltimore, MA, USA

7. Mitchell GF (2009) Arterial stiffness and wave reflection: biomarkers of cardiovascular. Artery Res 3(2):56-64

8. Johansson RS, Flanagan JR (2009) Coding and use of tactile signals from the fingertips in object manipulation tasks. Nature 10:345-359

9. Serina ER, Mockensturm E, Mote CDJ, Rempel D (1998) A structural model of the forced compression of the fingertip pulp. J Biomech 31(7):639-646

10. Wu JZ, Dong RG, Rakheja S, Schopper AW, Smutz WP (2004) A structural fingertip model for simulating of the biomechanics of tactile sensation. Med Eng Phys 26:165-175

11. Zimmer SM (2008) Assessment and initial care of fingertip and nailbed injuries. JUCM 3(2):11-17

12. Luo YD, Shen YT, Mohan N (2013) Durable and cost-effective 3-D microforce sensor for musical tuning enhanced micro palpation of 
biological entities. In: Proceedings of the 2013 IEEE international conference on Sensors, 928-931, Baltimore, MA, USA

13. Murgo JP, Westerhof N, Giolma JP, Altobelli SA (1980) Aortic input impedance in normal man: relationship to pressure wave forms. Circulation 62:105-116

14. Kelly R, Hayward C, Avolio A, O'Rourke M (1989) Non-invasive determination of age-related changes in the human arterial pulse. Circulation 80:1652-1659

15. Takazawa K, Tanaka N, Takeda K, Kurosu F, Ibukiyama C (2005) Underestimation of vasodilator effects of nitroglycerin by upper limb blood pressure. Hypertension 26(3):520-523

16. Fetics B, Nevo E, Chen CH, Kass DA (1999) Parametric model derivation of transfer function for noninvasive estimation of aortic pressure by radial tonometry. IEEE Trans Biomed Eng 46:698-706

17. Chen CH, Nevo E, Fetics B, Pak PH, Yin FC, Maughan WL, Kass DA (1997) Estimation of central aortic pressure waveform by mathematical transformation of radial tonometry pressure: validation of generalized transfer function. Circulation 95:1827-1836

18. Millasseau SC, Patel SJ, Redwood SR, Ritter JM, Chowienczyk PJ (2003) Pressure wave reflection assessed from the peripheral pulse is a transfer function necessary? Hypertension 41:1016-1020

19. Sugawara J, Komine H, Hayashi K, Maeda S, Matsuda M (2007) Relationship between augmentation index obtained from carotid and radial artery pressure waveforms. J Hypertens February (2):375-381

20. Kohara K, Tabara Y, Oshiumi A, Miyawaki Y, Kobayashi T, Miki T (2005) Radial augmentation index: a useful and easily obtainable parameter for vascular aging. America J Hypertens 18(S1):11-14

21. Millasseau SC, Kelly RP, Ritter JM, Chowienczyk PJ (2002) Determination of age-related increases in large artery stiffness by digital pulse contour analysis. Clin Sci 103:371-377

22. Salvi P (2012) Pulse waves: how vascular hermodynamics affects blood pressure. Springer, Milan

doi:10.1186/s40638-014-0019-y

Cite this article as: Luo et al:: Bioinspired non-invasive radial pulse sensor: from biomimetic design, system calibration, to clinic application. Robotics and Biomimetics 2014 1:19.

\section{Submit your manuscript to a SpringerOpen ${ }^{\circ}$ journal and benefit from:}

- Convenient online submission

- Rigorous peer review

- Immediate publication on acceptance

- Open access: articles freely available online

- High visibility within the field

- Retaining the copyright to your article 